From the Unità Operativa Ematologia 1, Dipartimento di Ematologia e Oncologia, Ospedale Maggiore, I.R.C.C.S; Divisione di Medicina I, Ospedale "Fatebenefratelli e Oftalmico"; Divisione di Ematologia, Ospedale Niguarda, Cà Granda, Milan; Dipartimento di Medicina Interna e Oncologia Medica, Università degli Studi, Policlinico S. Matteo, I.R.C.C.S., Pavia; Divisione di Ematologia, Ospedali Riuniti, Bergamo; Dipartimento di Ematologia, Azienda Ospedaliera Bianchi-MelacrinoMorelli, Reggio Calabria; Dipartimento di Oncologia e Ematologia, Centro Oncologico Modenese, Università degli Studi, Policlinico, Modena; Divisione di Ematologia, Ospedale S. Gerardo, Monza; Servizio di Ematologia, Arcispedale S. Maria Nuova, Reggio Emila, Italy; Clinical Hematology Service of Hématologie Clinique, Hôpital Schaffner, Lens, France.

Submitted June 22, 2004; accepted March 16, 2005

Supported by a grant (Ricerca Corrente, Progetti a Concorso) from the Italian Ministry of Health to Ospedale Maggiore IRCCS, Milan, Italy.

A complete list of the Gruppo Italiano Studio Linfomi (GISL) Centres who referred patients to the study is given at the end of this article.

Authors' disclosures of potential conflicts of interest are found at the end of this article.

Address reprint requests to Luca Baldini, MD, Unità Linfomi/Mielomi, Unità Operativa Ematologia 1, Centro G. Marcora, Ospedale Maggiore, I.R.C.C.S., Via F. Sforza 35, 20122 Milano, Italy; e-mail: lubaldini@ policlinico.mi.it.

๑ 2005 by American Society of Clinical Oncology

0732-183X/05/2321-4662/\$20.00

DOI: $10.1200 / J C O .2005 .06 .147$

\title{
Immunoglobulin M Monoclonal Gammopathies of Undetermined Significance and Indolent Waldenström's Macroglobulinemia Recognize the Same Determinants of Evolution Into Symptomatic Lymphoid Disorders: Proposal for a Common Prognostic Scoring System
}

Luca Baldini, Maria Goldaniga, Andrea Guffanti, Chiara Broglia, Sergio Cortelazzo, Andrea Rossi, Enrica Morra, Mariangela Colombi, Vincenzo Callea, Enrico Pogliani, Fiorella Ilariucci, Stefano Luminari, Pierre Morel, Giampaolo Merlini, and Paolo Gobbi

\section{$\begin{array}{llllllll}\text { A } & \text { B } & \text { S } & \text { T } & \text { R } & \text { A } & \text { C } & T\end{array}$}

\section{Purpose}

To evaluate the clinicohematologic variables at diagnosis that are prognostically related to neoplastic progression in patients with immunoglobulin $\mathrm{M}(\mathrm{lg} \mathrm{M})$ monoclonal gammopathies of undetermined significance (MGUS), and indolent Waldenström's macroglobulinemia (IWM), and propose a scoring system to identify subsets of patients at different risk.

\section{Patients and Methods}

We evaluated 217 patients with IgM MGUS and 201 with IWM (male-female ratio, 131:86 and 117:84; mean age, 63.7 and 63.6 years, respectively) diagnosed on the basis of serum monoclonal component (MC) levels and bone marrow lymphoplasmacytic infiltration degree. The variables selected by univariate analyses were multivariately investigated; on the basis of their individual relative hazards, a scoring system was devised to identify subsets of patients at different risk of evolution.

\section{Results}

After a median follow-up of 56.1 and 60.2 months, 15 of 217 MGUS and 45 of 201 IWM patients, respectively, required chemotherapy for symptomatic WM (13 and 36), nonHodgkin's lymphoma ( 2 and 6 ) and amyloidosis ( 0 and 3 ). The median time to evolution (TTE) was not reached for MGUS and was 141.5 months for IWM. The variables adversely related to evolution were qualitatively the same in both groups: MC levels, Hb concentrations and sex. A scoring system based on these parameters identified three risk groups with highly significant differences in TTE in both groups $(P<.0001)$.

\section{Conclusion}

MGUS and IWM identify disease entities with different propensities for symptomatic neoplastic evolution. As both have the same prognostic determinants of progression, we propose a practical scoring system that, identifying different risks of malignant evolution, may allow an individualized clinical approach.

\section{J Clin Oncol 23:4662-4668. (C) 2005 by American Society of Clinical Oncology}

\section{INTRODUCTION}

The presence of a serum immunoglobulin $\mathrm{M}$ (IgM) monoclonal component (MC) is associated with a spectrum of lymphoid disorders including monoclonal gammo- pathies of undetermined significance (MGUS), lymphoplasmacytic lymphoma/Waldenström's macroglobulinemia (WM), B-cell non-Hodgkin's lymphomas (NHL; mainly low-grade histotypes), rare forms of IgM multiple myeloma/plasmacytoma (MM) 
and IgM-related disorders (IgM-RDs). ${ }^{1,2}$ IgM-RDs are conditions with symptoms that are mainly related to the specific anticorpal properties of MC (eg, anti-RBC, antiplatelet, antineural, and cryoglobulnemic activity). Because of their particular clinical presentations, these forms are often excluded from hematologic studies analyzing macroglobulinemias, but they are similar to IgM MGUS in terms of the absence of an underlying lymphoma and, as recently shown by Morra et $\mathrm{al}^{3}{ }^{3}$ a similar probability of transformation into a lymphoid malignancy. The most prevalent of the conditions whose main feature at presentation is the presence of serum MC are IgM MGUS and WM in its indolent or asymptomatic form. There is no clear consensus concerning their diagnostic criteria or the features at diagnosis that are prognostically related to progression to treatment-requiring disease. In accordance with the Second International Workshop on WM, WM is diagnosed when IgM MC (at whatever concentration) accompanies a bone marrow lymphoplasmacytic lymphoma. ${ }^{4}$ Other updated reviews of monoclonal gammopathy suggest more definite criteria based on serum MC levels and the degree of bone marrow infiltration..$^{5-7}$

Taking these proposals into account, we analyzed a large series of patients with IgM MGUS or indolent/asymptomatic WM (IWM) in order to verify whether the two diseases actually have distinct clinical courses, and to define the variables at diagnosis that are related to a neoplastic evolution requiring chemotherapy. We show that IgM MGUS and IWM seem to be different in terms of overall survival and the probability of neoplastic transformation, and that they both have the same determinants related to evolution. On the basis of these findings, we devised a scoring system to identify subsets of patients affected by asymptomatic macroglobulinemias (AM; IgM MGUS + IWM) at different risks of evolution. Better than the classification into IgM MGUS and IWM, which seems to be valid in defining the presence or absence of a lymphoma, this approach could offer a more precise definition of the risk of progression to a symptomatic and treatmentrequiring pathology.

\section{PATIENTS AND METHODS}

\section{Patients}

The study involved 418 patients with AM, and a minimum follow-up of 1 year who were diagnosed and followed up from June 1978, to January 2003, in 10 Italian institutions belonging to the Italian Lymphoma Study Group (Gruppo Italiano Studio Linfom [GISL]), in the Clinical Hematology Service of Hématologie Clinique, Hôpital Schaffner (Lens, France: 39 patients), in the Division of Hematology, Ospedale Maggiore (Bergamo, Italy: 101 patients), and in the Division of Hematology, Ospedale Niguarda (Cà Granda, Milan, Italy: 48 patients). The patients were subdivided into 217 with IgM MGUS and 201 with IWM according to previously reported criteria. ${ }^{5-7}$ The cutoff values were serum IgM MC levels $(<3$ or $\geq 3 \mathrm{~g} / \mathrm{dL})$ and/or the presence of more or less than $10 \%$ bone marrow infiltration by small lymphocytes showing plamacytoid/plasma cell differentiation, in absence of symptoms or related organ or tissue treatment-requiring impairment (ie, symptomatic cytopenia(s), and/or "B" symptoms and/or the progressive involvement of lymphopoietic organs and/or symptoms attributable to MC such as hyperviscosity syndrome, cryoglobulinemia, amyloidosis, or peripheral neuropathy). IgM MGUS was diagnosed when serum MC and bone marrow infiltration were less than $3 \mathrm{~g} / \mathrm{dL}$ and $10 \%$, respectively; IWM was diagnosed when serum $\mathrm{MC}$ was $\geq 3 \mathrm{~g} / \mathrm{dL}$ or bone marrow infiltration was $\geq 10 \%$. In our series, a serum MC level $\geq 3 \mathrm{~g} / \mathrm{dL}$ was detected in only 14 patients. Patients with IgM-producing MM, low grade B-cell NHL (other than the lymphoplasmacytic type), NHL in leukemic phase (clonal lymphocytes $\geq 5.0 \times 10^{9} / \mathrm{L}$ ), heavy chain disease, amyloidosis, and cases associated with hepatitis C virus (HCV) -related cryoglobulinemia or autoimmune-related macrogobulinemias (IgM-related disorders) were also excluded. Neoplastic evolution was defined as the appearance of clinical manifestations of WM or NHL, requiring treatment because of progressive marrow failure with development or worsening of anemia $(\mathrm{Hb}<2 \mathrm{~g} / \mathrm{dL}$ below the lower normal limit or $<10$ $\mathrm{g} / \mathrm{dL}$ ) and/or thrombocytopenia (PLT $\left.<100 \times 10^{9} / \mathrm{L}\right)$ and/or the presence of a serum $\mathrm{MC} \geq 5 \mathrm{~g} / \mathrm{dL}$ and/or a $\mathrm{MC}$ increase greater than $50 \%$ in less than 6 months, with the MC reaching at least a value of $3 \mathrm{~g} / \mathrm{dL}$ and/or the appearance of a lymphoid leukemic phase with a lymphocyte doubling time of less than 6 months and/or B symptoms and/or progressive splenomegaly and/or lymphadenopathy and/or symptoms attributable to MC (hyperviscosity syndrome, cryoglobulinemia, amyloidosis, or peripheral neuropathy).

\section{Laboratory and Clinical Studies}

In addition to routine laboratory tests (including the evaluation of serum lactate dehydrogenase and $\beta 2$ microglobulin levels and HCV antibody status), the type of M protein was defined by means of immunofixation, and quantified using serum protein electrophoresis and nephelometry. Serum polyclonal immunoglobulins were measured by means of nephelometry, and light chain proteinuria by means of cellulose acetate or gel-based electrophoresis. Under our conditions, the lowest detectable level of Bence-Jones proteinuria was $200 \mathrm{mg} / \mathrm{L}$. Unless otherwise required because of the appearance of symptoms or hematologic signs of progression, the routine laboratory tests and serum and urinary MC evaluations were performed three times a year for the first 2 years, and then every 6 months. A bone marrow aspirate was obtained at diagnosis from all of the patients; bone marrow biopsy was performed in 192 patients in different disease phases. Chest x-rays and abdominal ultrasonograms were taken every 12 to 24 months.

\section{Statistical Methods}

We comparatively evaluated the prognostic value of a series of clinical and laboratory parameters recorded at the time of the diagnosis of IgM MC in the two-patient subsets in relation to the clinical evolution of the disorder.

The main prognostic outcome was the time to evolution (TTE) to symptomatic lymphoproliferative disease, and was calculated from the date of diagnosis to that of clinical evolution. In addition to the basic epidemiological and clinical parameters, overall survival (OS; from the date of diagnosis to the date of death due to any cause or the date of the last clinical examination) was also taken into account in order to check the ultimate fate of the 
two patient populations. The TTE and OS curves were both calculated using the method of Kaplan and Meier. ${ }^{8}$

All of the clinical characteristics recorded at diagnosis were studied in relation to TTE in order to measure their ability to predict clinical evolution. To this end, a series of univariate regression analyses were first applied to the proportional hazards model to both groups of patients in order to assess the clinical variables related to TTE $\left(P<.05\right.$ was considered statistically significant). ${ }^{9}$ Subsequently, the selected variables were multivariately investigated in the same Cox model in order to obtain a hierarchical order using a stepwise technique ${ }^{10}$ to choose the best covariates independently related to TTE. By accounting the individual relative hazards of each covariate, their coefficients, the distribution of their values (continuous or discrete, such as sex), and of the limits of their variability (as imposed by the diagnostic criteria of $\mathrm{MC}$ ), a scoring system was devised to allow the simplest management of the essential clinical information. Finally, the scoring model was checked and validated in relation to TTE in both groups of patients.

\section{RESULTS}

The main demographic and hematological characteristics of IgM MGUS (217 patients) and IWM (201 patients) at diagnosis are presented in Table 1 . The patients' clinicohematologic features were not different except for the clinical parameters involved in their diagnostic discrimination (the percentage of bone marrow infiltration and, partially, serum MC concentration). After a median follow-up of 56.1 months (range, 12 to 242 months), corresponding to 1,176.8 person-years, 15 of the 217 patients with MGUS (6.9\%) required chemotherapy: 13 for symptomatic WM (five cases of anemia; one of B symptoms; one of splenomegaly; and six involving variable combinations of anemia, increased MC, macroglobulinemic purpura, B symptoms, and splenomegaly) and two for NHL (lymphoplasmacytic type). After a median follow-up of 60.2 months (range, 12 to 228 months) corresponding to 1,204.5 person-years, 45 (22.4\%) of the 201, patients with IWM required chemotherapy: 34 for symptomatic WM (11 cases of anemia, four of B symptoms, six of increased MC levels, one of macroglobulinemic purpura, and 12 involving more than one of these with or without splenomegaly), eight for NHL (four lymphoplamacytic, two diffuse large B-cell, one follicular, and one bronchial MALT lymphoma), and three for amyloidosis.

Figure 1A shows that OS was rather different in MGUS and IWM: median survival was not reached in either group, but there were respectively $91.8 \%$ and $73.5 \% 10$ - and 15 year survivors in the former group, and $80.9 \%$ and $62.1 \%$ in the latter. Figure $1 \mathrm{~B}$ shows that TTE at 10 and 15 years was respectively $85.5 \%$ and $75.1 \%$ among the MGUS patients, and $66.0 \%$ and $38.3 \%$ among the IWM patients.

The univariate analyses of the prognostic factors related to evolution in the two groups are shown in Table 2: the features that individually correlated with an evolution

\begin{tabular}{|c|c|c|}
\hline Variable & MGUS & IWM \\
\hline No. of patients & 217 & 201 \\
\hline \multicolumn{3}{|l|}{ Age, years } \\
\hline Mean & 63.7 & 63.6 \\
\hline SD & 11.3 & 10.0 \\
\hline Range & 29.9-89.3 & $35.8-86.1$ \\
\hline \multicolumn{3}{|l|}{ Male/female } \\
\hline No. & $131 / 86$ & $117 / 84$ \\
\hline Ratio & 1.52 & 1.39 \\
\hline Years of diagnosis & $1980-2003$ & $1978-2003$ \\
\hline \multicolumn{3}{|c|}{ Cases diagnosed before 1996} \\
\hline No. & 95 & 97 \\
\hline$\%$ & 44 & 48 \\
\hline \multicolumn{3}{|l|}{ Follow-up, months } \\
\hline Median & 56.1 & 60.2 \\
\hline Range & $12-242.1$ & $12-228.0$ \\
\hline \multicolumn{3}{|c|}{ Serum $M C$, densitometric value; $g / d L$} \\
\hline Mean & 0.88 & 1.57 \\
\hline$S D$ & 0.49 & 1.01 \\
\hline Range & $0.2-2.86$ & $0.2-3.90$ \\
\hline \multicolumn{3}{|c|}{$\begin{array}{l}\text { Serum IgM, nephelometric value; } \\
\mathrm{mg} / \mathrm{dL}\end{array}$} \\
\hline Mean & 885.8 & $1,665.9$ \\
\hline SD & 591.6 & $1,289.9$ \\
\hline Range & $210-2,990$ & $250-4,900$ \\
\hline$\kappa / \lambda$ ratio of serum $\mathrm{MC}$ & 4.18 & 3.51 \\
\hline \multicolumn{3}{|c|}{$\begin{array}{l}\% \text { bone marrow infiltration, } \\
\text { aspirate }\end{array}$} \\
\hline Mean & 6.6 & 24.0 \\
\hline $\mathrm{SD}$ & 2.3 & 14.9 \\
\hline Range & $2-10$ & $10-80$ \\
\hline \multicolumn{3}{|l|}{ Hemoglobin, g/dL } \\
\hline Mean & 14.0 & 13.5 \\
\hline SD & 1.5 & 1.4 \\
\hline Range & $10.1-17.9^{*}$ & $10.4-16.3$ \\
\hline \multicolumn{3}{|l|}{ Serum albumin, $d / d L$} \\
\hline Mean & 4.29 & 4.35 \\
\hline SD & 0.47 & 0.48 \\
\hline Range & $2.65-5.50$ & $3.00-5.40$ \\
\hline \multicolumn{3}{|l|}{ Serum creatinine, $\mathrm{mg} / \mathrm{dL}$} \\
\hline Mean & 0.95 & 0.98 \\
\hline SD & 0.19 & 0.25 \\
\hline Range & $0.30-1.50$ & $0.34-2.40$ \\
\hline \multicolumn{3}{|c|}{ Serum $\beta 2$ microglobulin, $\mathrm{mg} / \mathrm{L} \dagger$} \\
\hline Mean & 2.511 & 2.796 \\
\hline SD & 0.840 & 1.448 \\
\hline Range & $0.577-5.750$ & $0.800-10.385$ \\
\hline
\end{tabular}

Abbreviations: IgM, immunoglobulin M; MGUS, monoclonal gammopathies of undetermined significance; IWM, indolent Waldenström's macroglobulinemia; SD, standard deviation; MC, monoclonal component. *14 patients had $\mathrm{Hb}$ value between 10.1 and $12 \mathrm{~g} / \mathrm{dL}$ and serum MC $<2.0 \mathrm{~g} / \mathrm{dL}$ (12 patients $<1.5 \mathrm{~g} / \mathrm{dL})$.

+Values available for 104 of 217 IgM-MGUS and 110 of 201 IWM patients, normalized to $3.0 \mathrm{mg} / \mathrm{L}$ as the upper normal limit.

to symptomatic disease $(P \leq .05)$ were male sex, the densitometric serum MC value and hemoglobin concentration in the MGUS patients, and the same plus the nephelometric serum IgA value and (with a borderline level of statistical 

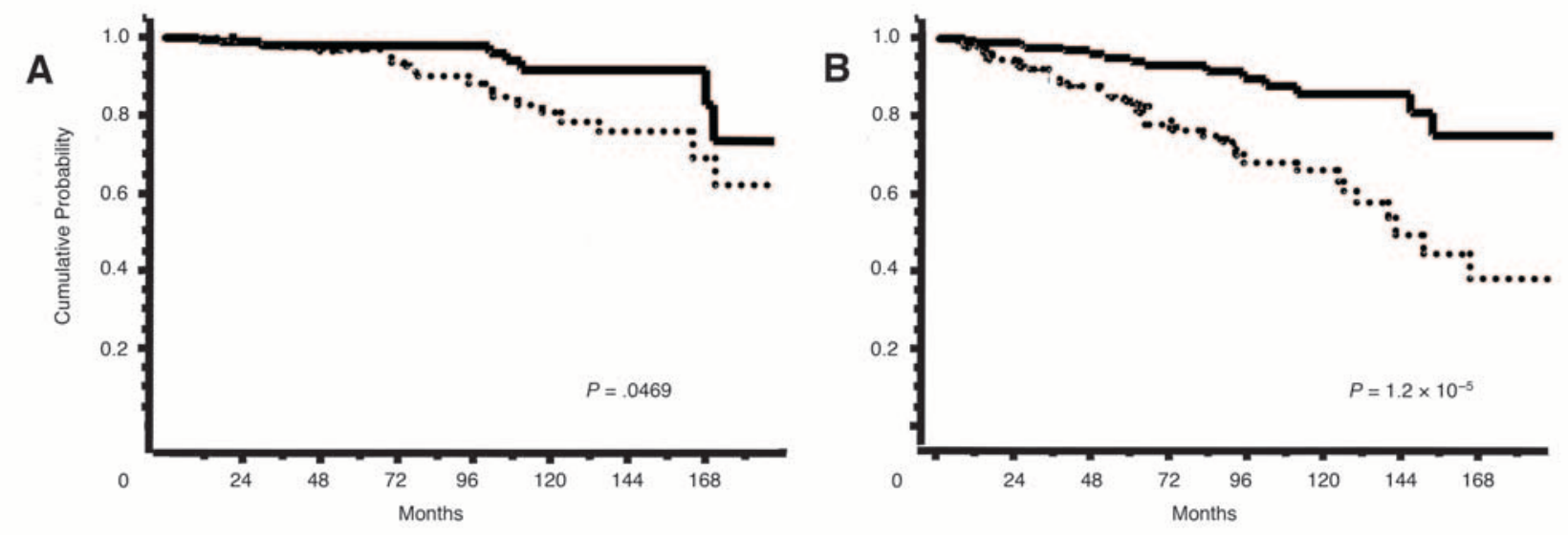

Fig 1. Overall survival (A) and time to evolution (B) in monoclonal gammopathies of undetermined significance (217 patients) and indolent Waldenström's macroglobulinemia (201 patients) groups (-, MGUS; $\cdots$, IWM).

significance) the percentage of bone marrow infiltration in the IWM patients.

Cox's multivariate analysis was used to evaluate the prognostic role of the variables emerging from the univariate study, and a stepwise technique selected the statistically best covariates independently related to TTE. Interestingly, as shown in Table 3 , the variables adversely related to evolution remained the same in both groups: male sex, densitometric serum MC concentration and hemoglobin level. Males with MGUS and IWM, respectively, had a 4.182 and 2.061 relative risk of evolution in comparison with females, which is a small variation considering that sex was treated as a nominal covariate. In relation to the other covariates, any

Table 2. Prognostic Role of Clinicohematological Variables at Diagnosis in Neoplastic Evolution: Univariate Analyses Made Separately of the Group of 217 Patients With IgM- MGUS and the 201 With IWM

\begin{tabular}{lrr}
\multicolumn{1}{c}{ Variables } & \multicolumn{2}{c}{$\begin{array}{c}\text { PValue of the } \\
\chi^{2} \text { Test }\end{array}$} \\
\cline { 2 - 3 } & MGUS & IWM \\
\hline Serum MC (densitometric value), g/dL & .0015 & $<.0001$ \\
Serum IgM (nephelometric value), $\mathrm{mg} / \mathrm{dL}$ & .2441 & $<.0001$ \\
Hemoglobin, g/dL & .0295 & .0240 \\
Percentage of bone marrow infiltration & .1127 & .0713 \\
Sex (M V F) & .0301 & .0261 \\
Serum IgA (nephelometric value), $\mathrm{mg} / \mathrm{dL}$ & .7213 & .0102 \\
Serum LDH, U/L & .6515 & .1132 \\
Serum $\beta 2$ microglobulin, $\mathrm{mg} / \mathrm{L}^{*}$ & .2601 & .3650 \\
Age, years & .9988 & .1447 \\
\hline
\end{tabular}

Abbreviations: IgM, immunoglobulin M; MGUS, monoclonal gammopathies of undetermined significance; IWM, indolent Waldenström's macroglobulinemia; MC, monoclonal component; $\mathrm{M}$, male; F, female; IgA, immunoglobulin A; LDH, lactate dehydrogenase.

*Values available for 104 of 217 MGUS and 110 of 201 IWM patients, normalized to $3 \mathrm{mg} / \mathrm{L}$ as the upper normal limit. unit decrease in serum hemoglobin multiplied the risk of evolution by a very similar factor ( 0.725 in MGUS patients and 0.685 in IWM patients), whereas densitometric serum $\mathrm{MC}$ concentration had rather different prognostic power in the two groups (an increase of $1 \mathrm{~g} / \mathrm{dL}$ in serum MC was associated with a relative risk of 3.995 in MGUS and 1.340 in IWM), although the distribution in the groups may not be considered free because a serum level of $\geq 3 \mathrm{~g} / \mathrm{dL}$ by definition allocated patients to the IWM group (Table 3 ).

On the basis of their common prognostic determinants of evolution and the similar individual relative hazards shown by at least two thirds of the significant covariates, we

\begin{tabular}{|c|c|c|c|}
\hline Covariates & Coefficient & $\begin{array}{c}P \\
\left(\chi^{2}\right)\end{array}$ & $\begin{array}{c}\text { Relative } \\
\text { Hazard } \\
\text { Ratio }\end{array}$ \\
\hline \multicolumn{4}{|l|}{ MGUS } \\
\hline $\begin{array}{l}\text { Serum } M C \text { (densitometric } \\
\text { value), } g / d L\end{array}$ & 1.385 & .0098 & 3.995 \\
\hline Hemoglobin, g/dL & -0.322 & .0325 & 0.725 \\
\hline $\operatorname{Sex}(M \vee F)$ & 1.431 & .0311 & 4.182 \\
\hline \multicolumn{4}{|l|}{ IWM } \\
\hline $\begin{array}{l}\text { Serum MC (densitometric } \\
\text { value), } g / d L\end{array}$ & 0.292 & .0150 & 1.340 \\
\hline Hemoglobin, g/dL & -0.378 & .0022 & 0.685 \\
\hline $\operatorname{Sex}(M \vee F)$ & 0.723 & .0442 & 2.061 \\
\hline
\end{tabular}

NOTE. Only the variables with a statistically significant level $(<0.05)$ at the preliminary univariate analysis were selected (ie, serum MC, hemoglobin and sex in both groups, with the addition of the percentage of marrow infiltration and serum IgA concentration in the IWM group). Abbreviations: IgM, immunoglobulin M; MGUS, monoclonal gammopathies of undetermined significance; IWM, indolent Waldenström's macroglobulinemia; MC, monoclonal component; $M$, male; F, female; LDH, lactate dehydrogenase. 
tried to devise a common scoring system for both clinical entities using the three significant factors they shared. In order to simplify its clinical use, cutoff levels of the quantitative variables were chosen to allow the best and simplest discrimination of patients at different risk of evolution. The scoring system is shown in Table 4 . The application of the system seems to divide the 217 MGUS and the 201 IWM patients into three risk groups (low, 0 to 2; intermediate, 3 to 5; and high, 6 to 9), which were associated with highly significant differences in TTE (Fig 2). The prognostic discrimination was very satisfactory in both groups despite the small number of events in the MGUS group, and there was a remarkably unbalanced prevalence of patients with unfavourable scores ( $\geq 6$, group 3): $14 \%$ in the MGUS group and $34 \%$ in the IWM group.

\section{DISCUSSION}

As recently codified in the Second International Workshop on Waldenström's Macroglobulinemia (held in Athens, Greece, in September 2002), IgM monoclonal gammopathies essentially consist of four clinical entities: IgM MGUS, IgM RDs, IWM, and symptomatic/treatment-requiring WM. ${ }^{4}$ When analyzing the asymptomatic forms, one particularly important aspect is to identify at diagnosis the variables capable of predicting different risks of their evolution to symptomatic WM. This is important not only in terms of prognosis communications with patients, but also in terms of follow-up planning. Kyle et $\mathrm{al}^{7}$ found that the relative risk of progression to lymphoma or a related disorder was 16-fold higher in IgM MGUS patients than in the general population, and that it was correlated with initial serum MC and albumin levels. In a series of patients with generically defined asymptomatic macroglobulinemias, Morra et al found that serum IgM levels, hemoglobin concentrations, and the presence of absolute lymphocytosis independently predicted a malignant evolution, ${ }^{11}$ and the same authors found that only MC levels of more than $3 \mathrm{~g} / \mathrm{dL}$ and hemoglobin levels of $\leq 12.5 \mathrm{~g} / \mathrm{dL}$ adversely correlated with progression to symptomatic disease in 27 cases of smouldering WM. ${ }^{12}$ When evaluating the frequency and

\begin{tabular}{|lcccc}
\hline $\begin{array}{c}\text { Table 4. Cut-Off Levels for a Prognostic Score, Drawn From the Best } \\
\text { Fitting Covariates and Their Relative Hazards As Shown in Table 3 }\end{array}$ \\
\cline { 2 - 6 } & \multicolumn{5}{c}{ Score } \\
\hline \multicolumn{6}{c}{ Covariate } & 0 & 1 & 2 & 3 \\
\hline $\begin{array}{c}\text { Serum monoclonal } \\
\text { component, g/dL }\end{array}$ & $<0.7$ & $0.7-1.399$ & $1.4-2.099$ & $\geq 2.1$ \\
$\begin{array}{l}\text { Hemoglobin, g/dL } \\
\text { Sex }\end{array}$ & $\geq 15$ & $13-14.99$ & $11-12.99$ & $<11$ \\
\hline
\end{tabular}

NOTE. Low risk: 0-2; intermediate risk: 3-5; high risk: 6-9. natural history of IWM cases, Alexanian et al found that hemoglobin and $\beta 2$ microglobulin levels correlated with early progression to symptomatic WM. ${ }^{13}$ Morra et al recently evaluated the prognostic features of two groups of patients affected by asymptomatic macroglobulinemias or IgM RDs, and concluded that although these forms are distinct clinical entities, they have a similar probability of transformation into lymphoid malignancy, and that hemoglobin levels and the erythrocyte sedimentation rate at diagnosis were of major statistical importance. ${ }^{3}$

In our study, we evaluated the prevalence of symptomatic neoplastic evolution in a large series of patients with asymptomatic macroglobulinemias divided into IgM MGUS and IWM forms on the basis of serum MC level and lymphoplasmacytic marrow infiltration, according to the previously reported criteria, ${ }^{5-7}$ and identified the features at diagnosis that are related to clinical progression. On the basis of OS and TTE, the two groups of patients were clearly distinct, thus confirming that the adopted diagnostic criteria seem to be useful and easily applicable. Patients with MGUS can therefore be reassured that they are not affected by an overt neoplastic disease, even though they need a specific clinical and laboratory examination.

It is interesting that both groups showed identical set of features at diagnosis that are prognostically related to a malignant and treatment-requiring evolution: sex, densitometric serum MC levels and hemoglobin concentrations. Being male had a negative prognostic impact: the males in both groups had an average three-fold higher relative risk of evolution in comparison with females, as result of the expected variability related to its nominal character observed in IgM MGUS group, and in the IWM one. Similarly, by integrating the coefficients and relative hazards obtained by multivariate analysis and the different variability of MC concentrations in MGUS and IWM, every $0.7 \mathrm{~g} / \mathrm{dL}$ increase in serum MC doubled the mean risk of evolution in both groups. It was calculated in the same manner that every $2 \mathrm{~g} / \mathrm{dL}$ decrease in hemoglobin doubled the risk of evolution.

These results partially confirm the observations of other authors, ${ }^{7,12,13}$ particularly in terms of the prognostic role of hemoglobin and serum MC concentrations. The highly negative prognostic impact of male sex on disease evolution has been previously described, but only in studies evaluating adverse prognostic variables for survival in patients with symptomatic macroglobulinemia. ${ }^{14,15}$ Serum $\beta 2$ microglobulin levels did not seem to predict disease evolution in our series, although their values at diagnosis were available in only half of the cases. However, a negative association between high serum $\beta 2$ microglobulin levels and worse survival has been documented in a series of symptomatic patients, and the same authors found that 57 concurrently evaluated cases with hemoglobin levels of $\geq 12 \mathrm{~g} / \mathrm{dL}$ and serum $\beta 2$ microglobulin levels of less than $3 \mathrm{mg} / \mathrm{L}$ had an excellent prognosis and a low probability 

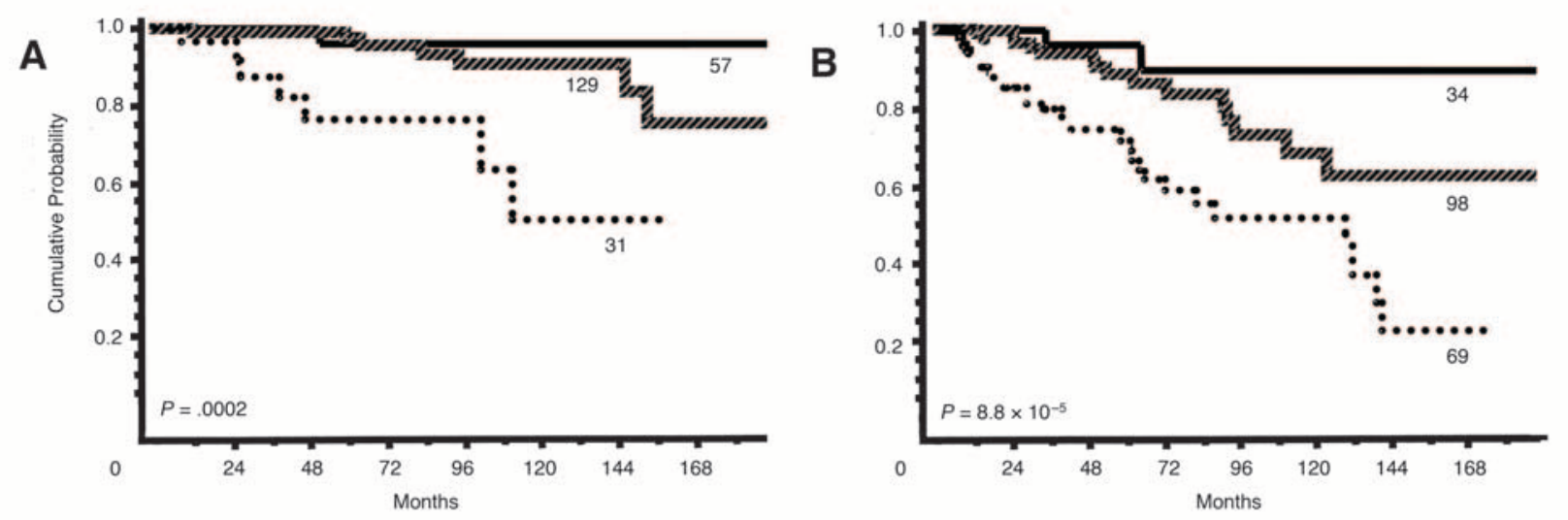

Fig 2. Time to evolution in monoclonal gammopathies of undetermined significance (A, 217 patients) and indolent-Waldenström's macroglobulinemia ( $B$, 201 patients) groups, according to proposed prognostic scoring system (-, low risk: 0-2 points; $\mathbb{Z}$, intermediate risk: $3-5$ points; $\cdots$, high risk: $\geq 6$ points).

of requiring therapy. ${ }^{16}$ Morra et $\mathrm{al}^{11}$ did not report any significant effect of $\beta 2$ microglobulin levels on disease progression. In relation to our series, $\beta 2$ microglobulin levels were evaluated in a subset of 214 patients, and were increased in 52 (24.3\%), including eight of 60 evolving cases for whom levels at diagnosis were available (three of 15 in the MGUS and five of 45 in the IWM group). This relatively small number of cases does not allow us to draw any conclusions, although it can be hypothesized that serum $\beta 2$ microglobulin may be a more useful prognostic marker in symptomatic than asymptomatic cases, as it is also supported by the relatively low frequency of increased levels in our series.

The similarity of the prognostic features (related with symptomatic evolution) in the two groups suggested the possibility of devising a single prognostic scoring system useful for all asymptomatic macroglobulinemias because the different evolutionary rate observed in MGUS and IWM could be largely explained by the different prevalence of patients with unfavourable evolution-related prognostic factors within the groups. From this point of view, a simple prognostic scoring system may be a useful and complementary way of better defining clinical conditions with a different tumor burden whose diagnostic definition is still debated. ${ }^{1,2,4,14,16-25}$ The Atene Workshop on WM was also unable to propose clear and reproducible diagnostic criteria for macroglobulinemias, particularly in the case of borderline situations that may be considered different clinical presentations of the same spectrum of lymphoid disorders. Taking this into account, we developed a simple prognostic scoring system that made it possible to divide the whole series into three groups at low (score, 0 to 2), intermediate (score, 3 to 5), or high risk of malignant progression (score, 6 to 9) on the basis of their significantly different TTE. The observation that the patients destined to evolve were more frequent in IWM group than in the MGUS one, and that the evolution-related variables were the same for both groups, suggests that these two subsets of macroglobulinemia may belong to a continuum of nosological entities probably mainly characterized by the absence of symptoms.

The well-established categorization of IgM MGUS and IWM helps physicians in their general approach to patients and in communicating clinical information (there is or is not an underlying overt lymphoma), but the proposed prognostic scoring system could provide additional information about the risk of evolution, and the application of a combined approach could be a more personalized means of simplifying patient management (eg, making it possible to differentiate follow-up clinical examinations on the basis of the individual risk of evolution and select the patients who may benefit from the early administration of new drugs). In other words, we tried to make up for the lack of exhaustive diagnostic criteria by means of an approach capable of predicting different risks of progression to a symptomatic lymphoid disorder that is considered a pivotal phase in the clinical course.

In conclusion, we suggest that the first step in approaching clonal macroglobulinemias is to identify the presence or otherwise of treatment-requiring symptoms; in the case of asymptomatic forms, we confirm the usefulness of the well-established categories of MGUS and WM (in an indolent phase) in identifying an overt lymphoproliferative disorder. In addition to establishing the features at diagnosis related to malignant progression in the two diseases, we propose a practical clinical scoring system capable of identifying conditions at different risk of malignant evolution that may require an individualized clinical approach.

\section{Acknowledgment}

We thank Kevin Smart of LINK s.r.l., Milan, for his assistance in preparing the text. 


\section{Appendix}

The other participating centers were: Del Santo A., Bronzino B., Sezione di Oncologia, Ospedale S. Paolo, Milan, Italy; Cavanna L., Bertè R., Divisione di Medicina Oncologica ed Ematologia, Ospedale Civile, Piacenza, Italy; Iannitto E., Divisione di Ematologia, Università-Policlinico, Palermo;
Quarta G., Divisione di Ematologia, Ospedale A. Di Summa, Brindisi.

\section{Authors' Disclosures of Potential Conflicts of Interest}

The authors indicated no potential conflicts of interest.

\section{REFERENCES}

1. Kipps TJ: Waldenström macroglobulinemia, in Beutler E, Coller BS, Lichtman MA, et al (eds): Williams Hematology. New York, NY, McGraw-Hill, 2001, pp 1317-1325

2. Kyle RA, Rajkumar SV: Monoclonal gammopathies of undetermined significance, in Kyle RA, Gertz MA (eds): Monoclonal Gammopathies and Related Disorders: Hematology/Oncology Clinics of North America. Philadelphia, PA, W.B. Saunders Company, 1999, pp 1181-1202

3. Morra E, Cesana C, Klersy C, et al: Clinical characteristics and factors predicting evolution of asymptomatic IgM monoclonal gammopathies and IgM-related disorders. Leukemia 18: 1512-1517, 2004

4. Owen RG: Developing diagnostic criteria in Waldenström's macroglobulinemia. Semin Oncol 30:196-200, 2003

5. Kyle RA, Therneau TM, Rajkumar SV, et al: A long-term study of prognosis in monoclonal gammopathy of undetermined significance. N Engl J Med 346:564-569, 2002

6. The International Myeloma Working Group: Criteria for the classification of monoclonal gammopathies, multiple myeloma and related disorders: A report of the International Myeloma Working Group. Br J Haematol 121:749-757, 2003

7. Kyle RA, Therneau TM, Rajkumar SV, et al: Long-term follow-up of IgM monoclonal gammopathy of undetermined significance. Blood 102: 3759-3764, 2003

8. Kaplan EL, Meier P: Nonparametric estimation from incomplete observations. J Am Stat Assoc 53:457-481, 1958
9. Cox D: Regression model and life-table. J R Stat Soc 34:187-220, 1972

10. Armitage P, Berry G: Statistical Methods in Medical Research (ed 2). Oxford, United Kingdom, Blackwell Scientific Publications, 1987, pp 434-438

11. Morra E, Cesana C, Klersy C, et al: Predictive variables for malignant transformation in 452 patients with asymptomatic IgM monoclonal gammopathy: Hematology/Oncology Studies and Trials (HOST) Group, Italy. Semin Oncol 30:172177, 2003

12. Cesana $C$, Miqueleiz $S$, Bernuzzi $P$, et al: Smouldering Waldenström's macroglobulinemia: Factors predicting evolution to symptomatic disease. Semin Oncol 30:231-235, 2003

13. Alexanian $R$, Weber $D$, Delasalle $K$, et al: Asymptomatic Waldenstrom's macroglobulinemia. Semin Oncol 30:206-210, 2003

14. Morel $P$, Monconduit $M$, Jacomy $D$, et al: Prognostic factors, in Waldenström macroglobulinemia, a report on 232 patients with the description of a new scoring system and its validation on 253 other patients. Blood 96:852-858, 2000

15. Facon $T$, Brouillard $M$, Duhamel $A$, et al: Prognostic factors in Waldenström macroglobulinemia: A report of 167 cases. J Clin Oncol 11: 1553-1558, 1993

16. Dhodapkar M, Jacobson JL, Gertz A: Prognostic factors and response to fludarabine therapy in patients with Waldenström macroglobulinemia: Results of United States intergroup trial (Southwest Oncology Group S9003). Blood 98:41-48, 2001

17. Longo DL: Plasma cell disorders, in Fauci $A S$, Braunwald $E$, Isselbacher $K J$, et al (eds):
Harrison's Principles of Internal Medicine. New York, NY, McGraw-Hill, 1998, pp 712-718

18. Dimopuolos MA, Panayiotidis $P$, Moulopoulos LA, et al: Waldenström's macroglobulinemia: Clinical features, complications and management. J Clin Oncol 18:214-226, 2000

19. Gobbi PG, Bettini R, Montecucco C, et al: Study of prognosis in Waldenström macroglobulinemia: A proposal of simple binary classification with clinical and investigational utility. Blood 83:2939-2945, 1994

20. Garcia-Sanz R, Montoto S, Torrequebrada A, et al: Waldenström macroglobulinemia: Presenting features and outcome in a series with 217 cases. Br J Haematol 115:575-582, 2001

21. Kyle RA, Garton JP: The spectrum of monoclonal gammopathy in 430 cases. Mayo Clin Proc 62:719-731, 1987

22. Berger $F$, Isaacson PG, Piris MA, et al: Lymphoplasmacytic lymphoma/Waldenström macroglobulinemia, in Jaffe ES, Harris NL, Stein $\mathrm{H}$, et al (eds): Pathology and Genetics of Tumours of Haematopoietic and Lymphoid Tissues: World Health Organization Classification of Tumours. Lyon, France, IARC Press, 2001, pp 132-134

23. Lindstrom FD, Dashlstrom V: Multiple myeloma or benign monoclonal gammopathy? A study of differential diagnostic criteria in 44 cases. Clin Immunol Immunopathol 10:168-174, 1978

24. Mac Kenzie MR, Fudenberg HH: Macroglobulinemia: An analysis of forty patients. Blood 39:874-889, 1972

25. Moller-Petersen J, Schmidt EB: Diagnostic value of the concentration of M-component in initial classification of monoclonal gammopathy. Scand J Haematol 26:295-301,1986 